\section{Bizarre gastric ulcer related to betel quid use: report of two cases}

A 47-year-old gentleman, who had chewed 20 betel quids daily for 25 years, presented with a 3-day history of melena. Esophagogastroduodenoscopy (EGD) disclosed an ulcerated tumor $3 \mathrm{~cm}$ in size in the antrum ( $\bullet$ Fig. 1). Biopsy of the tumor revealed acute and chronic inflammation. The patient quit chewing betel quids. Because of a strong suspicion of malignancy, EGD was repeated 5 days later. It showed ulcers, from 0.5 to $2 \mathrm{~cm}$ in diameter, in the antrum. Biopsy again disclosed acute and chronic inflammation. Hence, betel-quid-related gastric injury was suspected. EGD 1 month later showed only a scar in the antrum ( $\bullet$ Fig. 2) and the histology again proved benign.

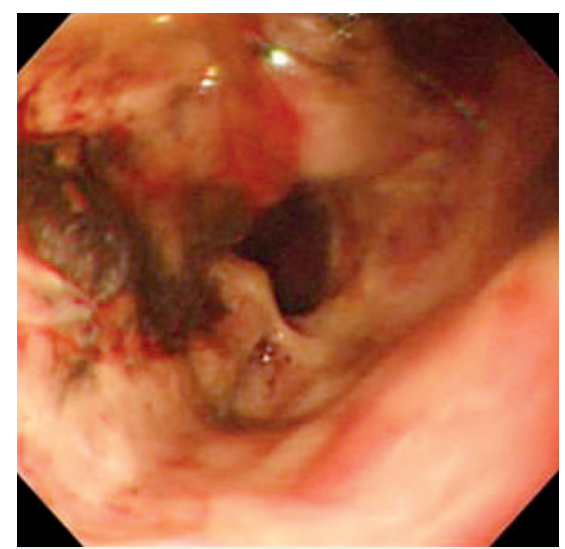

Fig. 1 Esophagogastroduodenoscopy (EGD) showed an ulcerated tumor $3 \mathrm{~cm}$ in size in the gastric antrum, involving the pyloric ring.

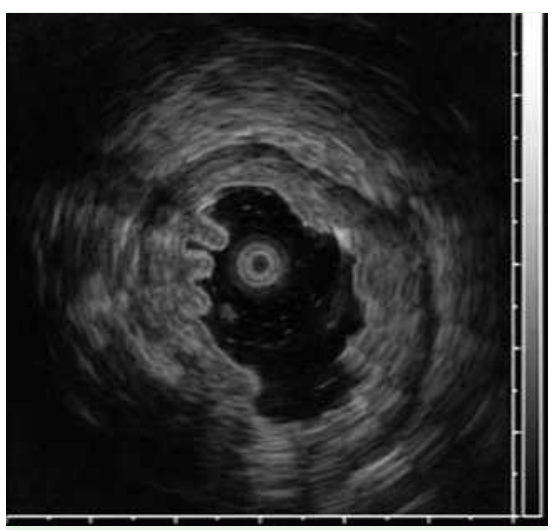

Fig. 4 Endoscopic ultrasound showed thickening of gastric antral wall, up to $10 \mathrm{~mm}$ in thickness.
Another 44-year-old gentleman, who had chewed 20 betel quids daily for 20 years, presented having produced coffeeground vomitus for 1 day. EGD showed ulcers from 0.2 to $3 \mathrm{~cm}$ in diameter in the antrum ( $\bullet$ Fig. 3). Biopsy showed acute and chronic inflammation. Helicobacter pylori was found. Endoscopic ultrasound revealed thickening of the antrum. The mucosa and submucosa planes were blurred, but the muscularis propria was intact ( $\bullet$ Fig. 4). The patient quit chewing betel quids and underwent Helicobacter pylori eradication therapy. EGD 2 months later revealed two tiny healing ulcers in the antrum ( Fig.5). Biopsy disclosed chronic inflammatory cells in the lamina propria. EGD 3 months later showed only

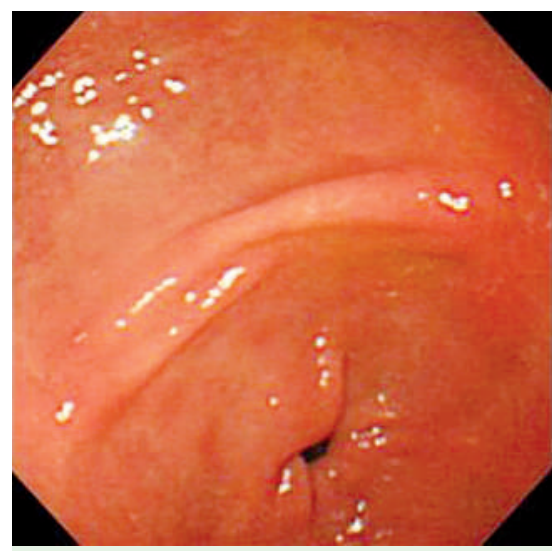

Fig. 2 EGD disclosed a small ulcer scar in the posterior wall of the gastric antrum.

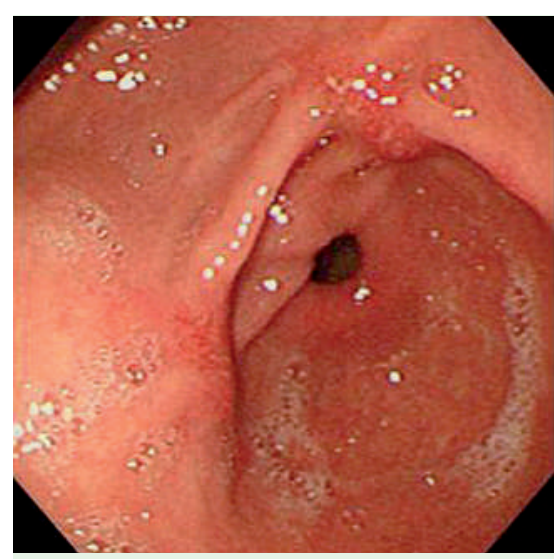

Fig. 5 EGD disclosed two tiny healing ulcers in the gastric antrum. erythematous changes of the antral mucosa (†ig. 6).

Betel quid consumption results in gastric ulcers in animal studies [1,2]; this is the first report of betel-quid-induced ulcer in human patients. There is no laboratory marker, endoscopic finding, or pathologic feature pathognomonic of betel-quid-induced ulcer. Image studies aid little in the diagnosis. In areas where betel quid chewing is endemic, patients with gastric ulcer or suspected cancer should be asked whether they have a habit of betel quid use. These two cases show that betelquid-related gastric ulcer can be bizarre in appearance and repeated biopsy is mandatory.

Endoscopy_UCTN_Code_CCL_1AB_2AD_3AC

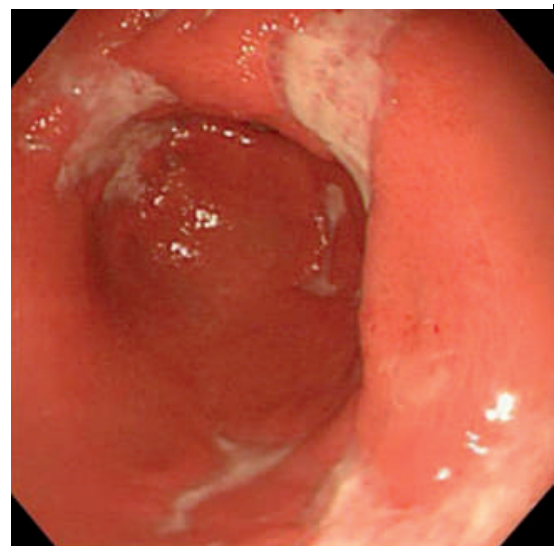

Fig. 3 EGD showed scattered shallow ulcers measuring from 0.2 to $3 \mathrm{~cm}$ in diameter in the gastric antrum.

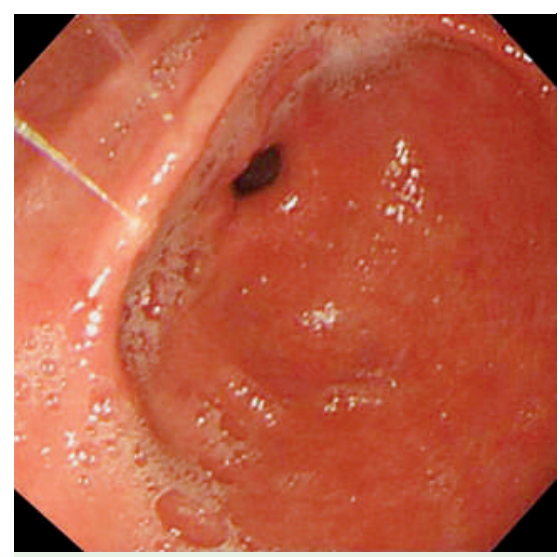

Fig. 6 EGD disclosed mildly erythematous changes of the mucosa in the gastric antrum. 
H. C. Lu' ${ }^{1}$, H. C. Huang ${ }^{1,2}$, A. F. Y. Li $\mathbf{i}^{2,3}$, H. C. Lin ${ }^{1,2}$, F. Y. Chang ${ }^{1,2}$, S. D. Lee ${ }^{1,2}$

${ }^{1}$ Department of Medicine, Taipei Veterans General Hospital, Taipei, Taiwan, Republic of China

2 National Yang-Ming University School of Medicine, Taipei, Taiwan, Republic of China

3 Department of Pathology, Taipei Veterans General Hospital, Taipei, Taiwan, Republic of China

\section{References}

1 Hung CR. Protective effects of lysozyme chloride and reduced glutathione on betel quid chewing-produced gastric oxidative stress and haemorrhagic ulcer in rats. Inflammopharmacology 2004; 12: 115-129

2 Hung CR, Wang PS. Role of histamine and acid back-diffusion in modulation of gastric microvascular permeability and haemorrhagic ulcers in betel-quid-fed rats. Inflammopharmacology 2004; 12: 277-287

Bibliography

DOI $10.1055 / s-2007-995684$

Endoscopy 2008; 40: E124-E125

(c) Georg Thieme Verlag KG Stuttgart · New York . ISSN 0013-726X
Corresponding author

\section{H. C. Huang, MD}

Division of Gastroenterology

Department of Medicine

Taipei Veterans General Hospital

No. 201, Sec. 2, Shih-Pai Road

Taipei 11217

Taiwan

Republic of China

Fax: + 886-2-28739318

hchuang2@vghtpe.gov.tw 